\title{
Quantification of Plasma or Serum Short-Chain Fatty Acids: Choosing the Correct Blood Tube
}

\author{
Lise Deroover $^{1}$, Eef Boets ${ }^{1}$, Yaxin Tie ${ }^{1}$, Greet Vandermeulen ${ }^{1}$ and Kristin Verbeke ${ }^{*_{1,2}}$ \\ ${ }^{1}$ Translational Research in Gastrointestinal Disorders, KU Leuven, Leuven, Belgium \\ ${ }^{2}$ Leuven Food Science and Nutrition Research Centre, KU Leuven, Leuven, Belgium
}

Received: July 12, 2017; Accepted: September 12, 2017; Published: November 11, 2017

*Corresponding author: Kristin Verbeke, Translational Research in Gastrointestinal Disorders, KU Leuven, Herestraat 49 Box 701 , Leuven 3000 , Belgium, Tel: +32 163301 50; Fax: +32 163307 23; E-mail: kristin.verbeke@kuleuven.be

\begin{abstract}
Background: Short-Chain Fatty Acids (SCFA; acetate, propionate and butyrate) are more and more recognised as mediators of local gut and systemic health. Quantification of SCFA in plasma and serum is challenging due to their low concentrations in human blood and the ubiquitous nature of acetate, requiring careful standardisation of the sample preparation procedure. Also the choice of the blood tube might affect the resulting concentrations.

Methods: SCFA concentrations were measured in blood samples $(10 \mathrm{~mL})$, collected from 10 healthy subjects in 7 different blood tubes. Control samples included milliQ (MQ) water and standard SCFA solutions. After preconcentration and clean-up of the samples using a hollow fiber liquid membrane extraction, SCFA concentrations were measured using Gas Chromatography (GC) coupled to Flame Ionisation Detection (FID).
\end{abstract}

Results: Acetate concentrations were significantly higher (ANOVA, p<0.01) when blood was collected in an EDTA K2 tube, where as propionate and/or butyrate levels were significantly higher in plasma prepared in a PST tube and a Barricor tube and serum prepared in a SST tube (ANOVA, $\mathrm{p}<0.01$ for all three tubes). Similar profiles of contamination were observed when analysing standard SCFA solutions that had been centrifuged in the different blood tubes. Lowest levels of contamination were observed when using red top glass serum tubes.

Conclusions: A red top glass serum tube is the preferred tube to collect blood for the quantification of SCFA. When plasma is preferred over serum, a lithium heparin tube is the most appropriate test tube.

Keywords: plasma/serum short-chain fatty acids; blood tube; polyacrylamide gel; gas chromatography; flame ionisation detection.

\section{Introduction}

Short-Chain Fatty Acids (SCFA; acetate, propionate and butyrate) are mainly produced by bacterial fermentation of undigested carbohydrates in the human colon [1]. They are considered as signaling molecules that provide a link between the diet, the microbiota and the host and mediate health benefits locally in the gut and on the systemic level $[2,3]$. Colonic-derived SCFA have been suggested to be involved in the prevention and treatment of metabolic syndrome, obesity, inflammatory bowel diseases and colon cancer [4-8]. However, these beneficial effects have mainly been demonstrated in in vitro experiments and animal models and need to be confirmed in human studies. The molecular mechanisms by which SCFA induce these effects are not completely understood and are the subject of many research. The fact that SCFA, in particular butyrate and to a lesser extent propionate inhibit histone deacetylases and in this way impact on the expression of genes with functions including cell proliferation, inflammation and apoptosis, may explain at least part of their effect [9]. In addition, SCFA are endogenous ligands for the G-protein coupled receptors 43 and 41 that have been found in the intestinal tract but also in adipose tissue, on immune cells, spleen and muscle cells. Activation of these receptors may be involved is the regulation of lipid and glucose metabolism [10].

Accurate quantification of SCFA in plasma and serum is essential to understand their role in mediating systemic effects. Colonic luminal SCFA concentrations, as well as fecal SCFA concentrations, are in the millimolar range and are relatively easy to quantify using gas chromatography after extraction into an organic solvent $[11,12]$. However, colonic SCFA are largely absorbed into the colonocytes where a part of the SCFA is used as an energy source and is immediately oxidized. The remaining SCFA are transported to the liver via the portal circulation where another (unknown) fraction is metabolized. Only the SCFA that pass the liver and escape splanchnic extraction end up in the peripheral circulation. As a result SCFA concentrations in plasma and serum are considerably lower than in the lumen of the colon and range between 50-100 $\mu \mathrm{mol} / \mathrm{L}$ for acetate and 0.5-10 $\mu \mathrm{mol} / \mathrm{L}$ for propionate and butyrate $[13,14]$. Because of these low concentrations, accurate analytical techniques are required. The precision of the measurements is further complicated by the ubiquitous nature of acetate, easily resulting in contaminations, which requires careful standardization of each step in the sample preparation [15]. In addition, the choice of blood collection tube may be important, as different blood tubes contain different additives, which may affect plasma and serum SCFA concentrations. For that reason, we investigated the influence of different blood collection tubes on plasma and serum SCFA concentrations. 


\section{Materials and Methods}

\section{Sample collection}

Blood samples from 10 healthy subjects (5 males and 5 females) were collected in 7 different blood tubes, i.e. an Ethylenediaminetetraacetic Acid (EDTA) K2 tube, a lithiumheparin tube, a plasma separator tube (PST), a Barricor tube, a red top glass serum tube, a red top glass serum tube to which $100 \mu \mathrm{L}$ of heparin (Heparine LEO, 100 U.I./ mL, Lier, Belgium) was added and a serum separator tube (SST) (BD Vacutainer ${ }^{\circledR}$, Erembodegem, Belgium). All tubes were centrifuged at 3000 $\mathrm{g}$ and $4^{\circ} \mathrm{C}$ for $10 \mathrm{~min}$. The resulting plasma or serum was transferred to a $2-\mathrm{mL}$ Eppendorf vial and stored at $-80^{\circ} \mathrm{C}$ until analysis. In addition, similar blood tubes were filled with $10 \mathrm{~mL}$ of Milli-Q® (MQ) water or $10 \mathrm{~mL}$ of a SCFA mix $(100 \mu \mathrm{mol} /$ Lacetate, $3 \mu \mathrm{mol} /$ Lpropionate and $1 \mu \mathrm{mol} /$ Lbutyrate) and centrifuged and stored as described above. Control MQ water and SCFA mix that have not been in contact with any blood tube were collected in a 2-mL Eppendorf vial and were also stored at $80^{\circ} \mathrm{C}$. This study was approved by the ethics committee of the University Hospital Leuven, Leuven, Belgium (Belgian Registration number: B322201732211) and all study procedures are in adherence to the Declaration of Helsinki. All participants signed written informed consent.

\section{Quantification of SCFA concentrations}

SCFA concentrations in plasma, serum, all MQ samples and all SCFA mix samples were measured using Gas Chromatography (GC) coupled to Flame Ionization Detection (FID) after preconcentration of the samples with hollow fiber supported liquid membrane extraction as described by Zhao et al. [16]. Briefly, a polypropylene hollow fiber tube (Accurel PP, model $50 / 280$, Wuppertal, Germany) was coated by dipping in a solution of Tri-n-octylphophine oxide in dihexyl ether (Sigma, St-Louis, USA) to form an organic liquid membrane on the pores of the fiber. Subsequently, the fiber was filled with $10 \mu \mathrm{L}$ of $0.15 \mathrm{~mol} / \mathrm{L} \mathrm{NaOH}$ and was inserted into a tube containing $100 \mu \mathrm{L}$ plasma or serum to which $20 \mu \mathrm{L}$ of $2 \mathrm{~mol} / \mathrm{L} \mathrm{HCl}$ and $50 \mu \mathrm{L}$ of internal standard (IS; $4 \mu \mathrm{mol} / \mathrm{L} 2$-ethyl butyrate and $20 \mu \mathrm{mol} / \mathrm{L} 3$-methyl valerate) had been added before dilution to $1.5 \mathrm{~mL}$. The tube was shaken for 16 h. Protonated SCFA diffuse into the fiber where they get ionized and remain trapped. In this way, SCFA were purified and up concentrated tenfold. After neutralization, the fiber content was analyzed on an Agilent 6890 N GC system equipped with a FID and an automatic liquid sampler. A fused-silica capillary column with Free Fatty Acid Phase (DB-FFAP 125-3237, J\&W Scientific, Agilent Technologies Inc., USA) of $30 \mathrm{~m} \times 0.53 \mathrm{~mm}$ I.D. coated with $0.5 \mu \mathrm{m}$ film thickness was used. The initial oven temperature was $100^{\circ} \mathrm{C}$ (3 min). Temperature was raised to $140^{\circ} \mathrm{C}(5 \mathrm{~min})$ by $4^{\circ} \mathrm{C} / \mathrm{min}$ and finally temperature was further raised to $235^{\circ} \mathrm{C}(5$ $\mathrm{min}$ ). Helium was supplied as the carrier gas in a constant flow mode of $4.2 \mathrm{~mL} / \mathrm{min}$. Glass wool was inserted into the glass liner of the splitless injection port. The FID and the injection port were heated to $240^{\circ} \mathrm{C}$ and $200^{\circ} \mathrm{C}$, respectively. The flow rates of hydrogen, air and nitrogen, as a make-up gas were 30, 300 and 20 $\mathrm{mL} / \mathrm{min}$, respectively. The total volume injected for GC analysis was $0.5 \mu \mathrm{L}$. The running time for each analysis contained 25.4 min. Data handling was carried out with Chemstation software (Revision B04.03, Agilent Technologies, USA).

Calibration curves were constructed using a standard SCFA solution. Blood samples from the 10 subjects were analyzed in triplicate, whereas MQ samples and SCFA mix samples were measured 6 times.

\section{Confirmation of SCFA purity using GC coupled to Mass Spectroscopy (MS)}

Serum from the same donor $(3 \mathrm{~mL})$ prepared either in a red top glass serum tube or in a SST tube was mixed with $3 \mathrm{~mL}$ of MQ water and $150 \mu \mathrm{L}$ of $0.15 \mathrm{~mol} / \mathrm{L} \mathrm{NaOH}$, centrifuged at 2000 $\mathrm{g}$ and $4^{\circ} \mathrm{C}$ for $20 \mathrm{~min}$ and deproteinized using Amicon Centriplus YM-30 centrifugal filters (30 kDa cut-off, Millipore Ltd., Watford, UK). After addition of $120 \mu \mathrm{L} 1 \mathrm{~mol} / \mathrm{L} \mathrm{NaOH}$, the filtrate was dried overnight in a vacuum concentrator (RVC 2-18, Christ, Osterhode am Harz, Germany) at $50^{\circ} \mathrm{C}$. SCFA were extracted in $400 \mu \mathrm{L}$ diethyl ether (Sigma-Aldrich, Steinheim, Germany) from the dried residue after addition of $100 \mu \mathrm{L} 4 \mathrm{~mol} / \mathrm{L} \mathrm{HCl}$. The ether layer was evaporated to $50 \mu \mathrm{L}$ after which $1 \mu \mathrm{L}$ of the extracted samples was injected on a GC-MS system (Trace 1300 and DSQ II XL, Thermo Electron Corporation, Waltham, MA, USA) with an Rxi ${ }^{\circledR}$-1ms column (Crossbond $囚 100 \%$ dimethyl polisyloxane; 30 m x 0,25 mm i.d. and $25 \mu \mathrm{m}$ film thickness; Restek, Bellefonte, Pennysylvania, USA). The initial oven temperature was $40^{\circ} \mathrm{C}(3$ $\min )$, then temperature was raised until $140^{\circ} \mathrm{C}$ at a rate of $4^{\circ} \mathrm{C} /$ min, followed by an increase to $240^{\circ} \mathrm{C}(6 \mathrm{~min})$ at a rate of $16^{\circ} \mathrm{C} /$ min. Helium was used as a carrier gas in a constant flow mode of $1 \mathrm{~mL} / \mathrm{min}$. The transfer line was maintained at $240^{\circ} \mathrm{C}$. The mass spectrometer was operated in full scan monitoring mode $(\mathrm{m} / \mathrm{z}$ $35-250$ ) and source temperature was set at $250^{\circ} \mathrm{C}$. Propionate and butyrate were identified by comparing the obtained mass spectrum with the NIST library (Gaithersburg, Maryland, USA). Butylated Hydroxytoluene (BHT), a solvent stabilizer present in diethyl ether was used as IS.

\section{Statistical analysis}

Results are presented as mean values with standard deviations (SD). Statistical analysis was performed with SPSS software (IBM SPSS Statistics 23, SPSS Inc., Chicago, Illinois, USA). Oneway ANOVA was performed followed by a post-hoc Tukey test. A p-value lower than 0.05 was considered statistically significant.

\section{Results}

\section{The influence of different blood collection tubes on SCFAconcentrations in plasma and serum}

Control MQ water contained very low amounts of acetate $(7.57 \pm 4.71 \mu \mathrm{mol} / \mathrm{L})$ and no detectable levels of propionate and butyrate indicating that the sample preparation protocol did not induce contamination Table 1 . After centrifugation in the different blood tubes, MQ watercontained higher levels of acetate, propionate and/or butyrate for all blood tubes compared to the control MQ water, except when centrifuged in a red top glass serum tube and a Barricor tube (Table 1). 
Table 1: SCFA concentrations in control MQ water and MQ water centrifuged in 7 different blood tubes ${ }^{1}$

\begin{tabular}{|c|c|c|c|}
\hline & Acetate & Propionate & Butyrate \\
\hline EDTA K2 tube $(\mu \mathrm{mol} / \mathrm{L})$ & $25.05 \pm 1.70^{\mathrm{a}}$ & $0.62 \pm 0.14^{\mathrm{a}}$ & $0.16 \pm 0.16^{\mathrm{a}}$ \\
\hline Lithium - Heparin tube $(\mu \mathrm{mol} / \mathrm{L})$ & $20.21 \pm 6.30^{\mathrm{ac}}$ & $0.19 \pm 0.25^{\mathrm{ac}}$ & $0.08 \pm 0.11^{\text {ad }}$ \\
\hline PST tube $(\mu \mathrm{mol} / \mathrm{L})$ & $13.33 \pm 2.89^{\mathrm{bc}}$ & $0.10 \pm 0.13^{\mathrm{ac}}$ & $0.41 \pm 0.09^{b}$ \\
\hline Barricor tube $(\mu \mathrm{mol} / \mathrm{L})$ & $10.53 \pm 5.84^{\mathrm{b}}$ & $0.17 \pm 0.22^{\mathrm{ac}}$ & $0.00 \pm 0.00^{\mathrm{ad}}$ \\
\hline Red top glass serum tube +Heparin $(\mu \mathrm{mol} / \mathrm{L})$ & $14.90 \pm 2.37^{\mathrm{bc}}$ & $0.29 \pm 0.41^{\mathrm{a}}$ & $0.13 \pm 0.01^{\mathrm{a}}$ \\
\hline Red top glass serum tube $(\mu \mathrm{mol} / \mathrm{L})$ & $8.18 \pm 3.03^{b}$ & $0.00 \pm 0.00^{c}$ & $0.00 \pm 0.00^{\mathrm{ad}}$ \\
\hline SST tube $(\mu \mathrm{mol} / \mathrm{L})$ & $11.96 \pm 2.14^{\mathrm{bc}}$ & $0.21 \pm 0.30^{\mathrm{ac}}$ & $0.74 \pm 0.15^{c}$ \\
\hline Control MQ water (no contact with tube; $\mu \mathrm{mol} / \mathrm{L}$ ) & $7.57 \pm 4.71^{b}$ & $0.00 \pm 0.00^{c}$ & $0.00 \pm 0.00^{\mathrm{d}}$ \\
\hline p-value & $6.00 \mathrm{E}-06$ & $4.29 \mathrm{E}-04$ & $1.21 \mathrm{E}-12$ \\
\hline
\end{tabular}

${ }^{1}$ Values are means $\pm \mathrm{SD} ; \mathrm{n}=6$. P-values are the result of a one-way ANOVA. $P<0.05$ was considered as statistically significant;

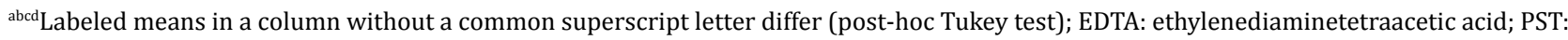
plasma separator tube; SST: serum separator tube; MQ: MilliQ.

Acetate concentrations were significantly higher when blood was collected in an EDTA K2 tube ( $p=0.047)$ whereas propionate and butyrate were significantly higher in a PST or a SST tube compared to the levels in the other blood tubes $\left(\mathrm{p}_{\text {propionate }}=0.024\right.$ and $\mathrm{p}_{\text {butyrate }}=0.002$ )(Table 2). When using a Barricor tube, only propionate levels were significantly increased compared to the other tubes (Table 2).

A SCFA mix with known concentrations of acetate, propionate and butyrate was analysed without prior contact with blood tubes resulting in concentrations of $99.45 \pm 6.46 \mu \mathrm{mol} / \mathrm{L}, 3.21 \pm$ $0.23 \mu \mathrm{mol} / \mathrm{L}$ and $1.07 \pm 0.05 \mu \mathrm{mol} / \mathrm{L}$, respectively, corresponding to the theoretical concentrations of $100 \mu \mathrm{mol} / \mathrm{L}, 3 \mu \mathrm{mol} / \mathrm{L}$ and 1 $\mu \mathrm{mol} / \mathrm{L}$. Higher acetate concentrations were found when the mix was centrifuged in an EDTA K2 tube. Similarly, concentrations of butyrate were higher when centrifuged in a PST and SST tube (Table 3). The levels of all three SCFA were lower in the SCFA mix after centrifugation in a red top glass serum tube to which heparin was added, compared to the concentrations in the SCFA mix as such, suggesting some loss of SCFA (Table 3 ).

\section{Identification of propionate and butyrate in serum centrifuged in a SST tube using GC-MS}

The concentrations of propionate and butyrate relative to BHT were respectively 4.5 and 9 times higher when the blood sample was centrifuged in a SST tube, compared to blood centrifuged in a red top glass serum tube (Table 4).

Analysis of the MS spectrum of the peaks with retention times corresponding to propionate and butyrate confirmed that the peaks were pure propionate (Figure 1a) and butyrate (Figure $1 \mathrm{~b}$ ),respectively, and ruled out the possibility of contamination with any co-eluting components.

Table 2: SCFA concentrations in blood centrifuged in 7 different blood tubes ${ }^{1}$

\begin{tabular}{|c|c|c|c|}
\hline & Acetate & Propionate & Butyrate \\
\hline \multicolumn{4}{|c|}{ Plasma } \\
\hline EDTA K2 tube $(\mu \mathrm{mol} / \mathrm{L})$ & $102.05 \pm 33.66^{\mathrm{a}}$ & $2.11 \pm 1.22^{\mathrm{a}}$ & $0.39 \pm 0.31^{\mathrm{a}}$ \\
\hline Lithium - Heparin tube $(\mu \mathrm{mol} / \mathrm{L})$ & $62.03 \pm 14.87^{\mathrm{b}}$ & $1.64 \pm 1.05^{\mathrm{a}}$ & $0.36 \pm 0.26^{a}$ \\
\hline PST tube $(\mu \mathrm{mol} / \mathrm{L})$ & $68.16 \pm 19.83^{\mathrm{b}}$ & $3.89 \pm 0.98^{\mathrm{b}}$ & $11.64 \pm 2.21^{\mathrm{b}}$ \\
\hline Barricor tube $(\mu \mathrm{mol} / \mathrm{L})$ & $76.88 \pm 19.70^{\mathrm{ab}}$ & $5.58 \pm 1.69^{c}$ & $0.70 \pm 0.52^{\mathrm{a}}$ \\
\hline Red top glass serum tube + Heparin $(\mu \mathrm{mol} / \mathrm{L})$ & $59.67 \pm 21.21^{b}$ & $1.56 \pm 0.51^{\mathrm{a}}$ & $0.38 \pm 0.18^{\mathrm{a}}$ \\
\hline \multicolumn{4}{|c|}{ Serum } \\
\hline Red top glass serum tube $(\mu \mathrm{mol} / \mathrm{L})$ & $62.25 \pm 26.91^{b}$ & $1.67 \pm 0.67^{\mathrm{a}}$ & $0.40 \pm 0.21^{\mathrm{a}}$ \\
\hline SST tube $(\mu \mathrm{mol} / \mathrm{L})$ & $63.24 \pm 19.28^{b}$ & $4.38 \pm 0.90^{\mathrm{bc}}$ & $16.99 \pm 1.99^{\circ}$ \\
\hline p-value & 0,001 & $2.66 \mathrm{E}-14$ & $8.37 \mathrm{E}-47$ \\
\hline
\end{tabular}

${ }^{1}$ Values are means \pm SD; $n=10$. P-values are the result of a one-way ANOVA. $P<0.05$ was considered as statistically significant; abcd Labeled means in a column without a common superscript letter differ (post-hoc Tukey test); EDTA: ethylenediaminetetraacetic acid; PST: plasma separator tube; SST: serum separator tube; MQ: MilliQ. 
Table 3: SCFA concentrations in SCFA mix centrifuged in 7 different blood tubes ${ }^{1}$

\begin{tabular}{|c|c|c|c|}
\hline & Acetate & Propionate & Butyrate \\
\hline EDTA K2 tube $(\boldsymbol{\mu m o l} / \mathbf{L})$ & $114.21 \pm 8.53^{\mathrm{ab}}$ & $3.04 \pm 0.14^{\mathrm{a}}$ & $1.05 \pm 0.09^{\mathrm{ab}}$ \\
\hline Lithium - Heparin tube $(\boldsymbol{\mu m o l} / \mathbf{L})$ & $95.27 \pm 13.18^{\mathrm{ab}}$ & $2.96 \pm 0.31^{\mathrm{ab}}$ & $0.99 \pm 0.08 \mathrm{ab}$ \\
\hline PST tube $(\boldsymbol{\mu m o l} / \mathbf{L})$ & $94.49 \pm 10.13^{\mathrm{ab}}$ & $3.08 \pm 0.30^{\mathrm{ab}}$ & $1.20 \pm 0.08^{\mathrm{bc}}$ \\
\hline Barricor tube $(\boldsymbol{\mu m o l} / \mathbf{L})$ & $118.02 \pm 26.97^{\mathrm{b}}$ & $3.67 \pm 0.73^{\mathrm{b}}$ & $0.99 \pm 0.14^{\mathrm{ab}}$ \\
\hline Red top glass serum tube $+\mathbf{H e p a r i n}(\boldsymbol{\mu m o l} / \mathbf{L})$ & $79.72 \pm 10.85^{\mathrm{a}}$ & $2.47 \pm 0.57^{\mathrm{ab}}$ & $0.97 \pm 0.06^{\mathrm{a}}$ \\
\hline Red top glass serum tube $(\boldsymbol{\mu m o l} / \mathbf{L})$ & $98.22 \pm 9.85^{\mathrm{ab}}$ & $3.07 \pm 0.23^{\mathrm{ab}}$ & $1.08 \pm 0.07^{\mathrm{ab}}$ \\
\hline SST tube $(\boldsymbol{\mu m o l} / \mathbf{L})$ & $92.87 \pm 8.80^{\mathrm{ab}}$ & $3.13 \pm 0.30^{\mathrm{ab}}$ & $1.45 \pm 0.06^{\mathrm{c}}$ \\
\hline Control SCFA mix (no contact with tube; $\boldsymbol{\mu m o l} / \mathbf{L})$ & $99.45 \pm 6.46^{\mathrm{ab}}$ & $3.21 \pm 0.23^{\mathrm{ab}}$ & $1.07 \pm 0.05^{\mathrm{ab}}$ \\
\hline $\boldsymbol{p}$-value & 0.047 & 0.024 & 0.002 \\
\hline
\end{tabular}

${ }^{1}$ Values are means $\pm \mathrm{SD} ; \mathrm{n}=6$. P-values are the result of a one-way ANOVA. $P<0.05$ was considered as statistically significant; ${ }^{\text {abcd }}$ Labeled means in a column without a common superscript letter differ (post-hoc Tukey test); EDTA: ethylenediaminetetraacetic acid; PST: plasma separator tube; SST: serum separator tube; MQ: MilliQ.

Table 4: Propionate and butyrate concentrations relative to BHT in a serum sample from a SST tube and a red top glass serum tube ${ }^{1}$

\begin{tabular}{|c|c|c|}
\hline & Propionate & Butyrate \\
\hline SST tube & $0.45 \pm 0.01$ & $0.26 \pm 0.01$ \\
\hline Red top glass serum tube & $0.10 \pm 0.02$ & $0.03+0.01$ \\
\hline
\end{tabular}

${ }^{1}$ Values are means \pm SD; $n=6$; SST: serum separator tube.

a
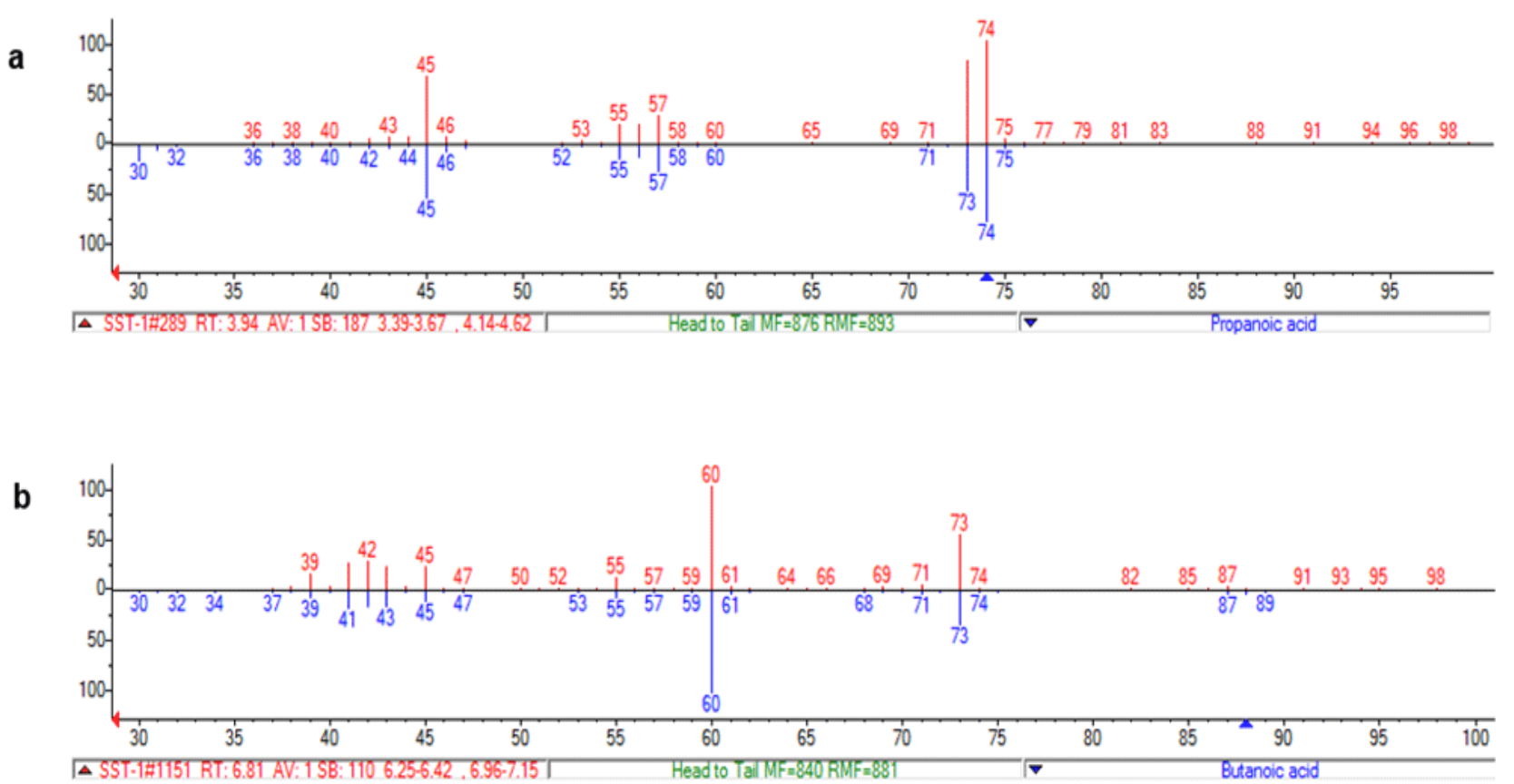

Figure 1: Mass spectrum of the propionate (a) and butyrate (b) peak detected in a serum sample centrifuged in a SST tube (blue) and the reference mass spectrum of propionate or butyrate in the NIST library (red). 


\section{Discussion}

Methods to quantify SCFA concentrations in biological fluids are often based on GC-MS or liquid chromatography coupled to MS. When GC is applied as separation technique, the SCFA are often extracted into an organic solvent with or without prior derivatization. Extraction of underivatized SCFA is often applied for the analysis of fecal SCFA but was, in our hands, not applicable to measure plasma and serum SCFA [17-20]. In particular for acetate and propionate, the extraction efficiency was low, which is evidenced from the relatively low octanol/water partitioning coefficients $(\log \mathrm{P}$ acetate $=0.2 ; \log \mathrm{P}$ propionate $=0.3$ and $\log$ $\mathrm{P}$ butyrate $=0.8$ ), and therefore not sufficiently reproducible. Prior derivatization of the SCFA with reagents such as propyl chloroformate or 1-(tert-butyldimethylsilyl)imidazole increases the extraction efficiency but also complicates the sample preparation protocol and increases the risk for contamination $[15,21]$. In a recently published paper, the need for extraction of underivatized SCFA was circumvented by direct injection of an acidified water concentrate on a GC-FID system. The samples were only preconcentrated using a hollow fiber supported liquid membrane extraction [16].

We applied this method to quantify SCFA in plasma and serum prepared from different blood tubes. Analysis of control MQ water that had not been in contact with blood tubes but underwent the sample preparation procedure indicated that induction of contamination due to the sample preparation was negligible.

Plasma and serum SCFA concentrations considerably varied depending on the type of blood tube used, possibly due to the presence of additives in the tubes. For example, the significantly higher acetate concentrations found in samples collected in EDTA K2 tubes are most likely due to residual acetate in the additive EDTA. Also when MQ water was centrifuged in those tubes, contamination with acetate was highest compared to the other tubes. Centrifugation of MQ in red top glass serum tubes did not induce any additional contamination compared to control MQ. To allow differentiation between contaminations with SCFA in test tubes with high concentrations versus loss of SCFA in test tubes with low concentrations, we analyzed the behavior of an aqueous SCFA mix with known concentrations of acetate, propionate and butyrate in the different test tubes. The differences between the different test tubes were in accordance with the differences observed when analyzing blood samples. The higher concentrations of acetate, particularly in the EDTA K2 tube, and of propionate and/or butyrate in the SST, PST and Barricor tube than the theoretically possible ones confirmed that the observed discrepancy was due to contamination rather than to loss of SCFA.

The significantly higher propionate and/or butyrate concentrations in plasma or serum obtained using a PST and Barricor tube or SST tube, respectively, are more difficult to explain. Both SST and PST tubes contain a polyacrylamide gel that separates plasma or serum from blood cells during centrifugation, facilitating in this way transfer of plasma or serum to another vial. Polyacrylamide is a linear or cross-linked synthetic polymer formed from acrylamide subunits and usually contains less than $0.05 \%$ of residual acrylamide monomer [22]. Some polyacrylamide polymers are copolymers of acrylamide and acrylic acid which results in a more ionic character of the polymer. GC-FID analysis does not yield structural information and hence, did not allow excluding contamination of the samples with acrylamide or acrylic acid. As those compounds are structurally related to propionate and butyrate, we hypothesized that they might co-elute from the analytical column during GC analysis. Using GC-MS and analysis of the MS spectra, we confirmed that the large peaks of propionate and butyrate in the serum from SST tubes only contained pure propionate and butyrate and excluded the possibility of co-eluting peaks.

It need to be mentioned that the polyacrylamide gel contains products from bovine origin. However, it is not specified whether these products are bovine plasma or serum. If this is the case, the differences in SCFA concentrations in blood centrifuged in a SST or PST tube can be explained by contaminations of our samples with bovine plasma or serum present in the gel.

The Barricor tube is a recently developed blood collection tube as an alternative to the PST tube. This tube contains an elastomer as mechanical separator instead of a polyacrylamide gel and should in this way interfere less with the tested analytes. Whether this elastomer contains propionate or not, is not tested and therefore not excluded.

When MQ water and the SCFA mix were centrifuged in a PST or a SST tube, the increase in propionate and butyrate concentrations compared to control MQ was less pronounced than the increment in PST plasma or SST serum compared to the tubes without gel. However, it needs to be mentioned that the gel in the PST and SST tube only migrates when centrifuging a whole blood sample. When these tubes are centrifuged with MQ water or SCFA mix, the gel is not activated and does not move, which might explain the lower increase in propionate and butyrate compared to control MQ or SCFA mix.

\section{Conclusions}

In conclusion, hollow fiber extraction of SCFA from plasma/ serum followed by GC-FID analysis allows reliable quantification of plasma/serum SCFA. The procedure as such does not induce any contamination, although the choice of blood tube affects the results. Red top glass serum tubes, not containing any additive, are the preferred tubes for SCFA quantification as they induce no contamination. When plasma is preferred over serum, a lithium heparin tube is the most appropriate test tube. EDTA K2 tubes yield significantly higher acetate concentrations, most likely due to residual acetate from the additive EDTA. Blood tubes that contain a polyacrylamide separator gel should be avoided as they induce significantly higher propionate and/or butyrate levels, possibly due to the bovine origin of compounds contained in the gel. Even the more inert elastomer in the Barricor tube induced propionate contamination and is not suitable for plasma SCFA analysis. 


\section{References}

1. Topping DL, Clifton PM. Short-chain fatty acids and human colonic function: roles of resistant starch and nonstarch polysaccharides. Physiol Rev. 2001;81(3):1031-1064.

2. Macfarlane S, Macfarlane GT. Regulation of short-chain fatty acid production. Proc Nutr Soc. 2003;62(1):67-72. doi: 10.1079/ PNS2002207

3. Koh A, De Vadder F, Kovatcheva-Datchary P, Backhed F. From dietary fiber to host physiology: short-chain fatty acids as key bacterial metabolites. Cell. 2016;165(6):1332-1345. doi: 10.1016/j. cell.2016.05.041

4. Woting A, Blaut M. The intestinal microbiota in metabolic disease. Nutrients. 2016;8(4):202. doi: 10.3390/nu8040202

5. Sun M, Wu W, Liu Z, Cong Y. Microbiota metabolite short chain fatty acids, GPCR, and inflammatory bowel diseases. J Gastroenterol. 2017;52(1):1-8. doi: 10.1007/s00535-016-1242-9

6. Macfarlane GT, Macfarlane S. Fermentation in the human large intestine: its physiologic consequences and the potential contribution of prebiotics. J Clin Gastroenterol. 2011;45 Suppl:S120-7. doi: 10.1097/MCG.0b013e31822fecfe

7. Canfora EE, Jocken JW, Blaak EE. Short-chain fatty acids in control of body weight and insulin sensitivity. Nat Rev Endocrinol. 2015;11(10):577-91. doi: 10.1038/nrendo.2015.128

8. Bultman SJ. Interplay between diet, gut microbiota, epigenetic events, and colorectal cancer. Mol Nutr Food Res. 2017;61(1): doi: 10.1002/ mnfr.201500902

9. Johnstone RW. Histone-deacetylase inhibitors: novel drugs for the treatment of cancer. Nat Rev Drug Discov. 2002;1(4):287-99. doi: $10.1038 / \operatorname{nrd} 772$

10.den Besten G, van Eunen K, Groen AK, Venema K, Reijngoud DJ, Bakker BM. The role of short-chain fatty acids in the interplay between diet, gut microbiota, and host energy metabolism. J Lipid Res. 2013;54(9):2325-2340. doi: 10.1194/jlr.R036012

11. Rahat-Rozenbloom S, Fernandes J, Gloor GB, Wolever TM. Evidence for greater production of colonic short-chain fatty acids in overweight than lean humans. Int J Obes. 2014;38(12):1525-1531. doi: 10.1038/ ijo.2014.46

12. Fernandes J, Su W, Rahat-Rozenbloom S, Wolever TM, Comelli EM. Adiposity, gut microbiota and faecal short chain fatty acids are linked in adult humans. Nutr Diabetes. 2014;4:e121. doi: 10.1038/ nutd. 2014.23
13. Priyadarshini M, Thomas A, Reisetter AC, Scholtens DM, Wolever TM, Josefson JL, et al. Maternal short-chain fatty acids are associated with metabolic parameters in mothers and newborns. Transl Res. 2014;164(2):153-157. doi: 10.1016/j.trsl.2014.01.012

14. Fernandes J, Vogt J, Wolever TM. Inulin increases short-term markers for colonic fermentation similarly in healthy and hyperinsulinaemic humans. Eur J Clin Nutr. 2011;65(12):1279-1286. doi: 10.1038/ ejcn.2011.116

15. Pouteau E, Meirim I, Metairon S, Fay LB. Acetate, propionate and butyrate in plasma: determination of the concentration and isotopic enrichment by gas chromatography/mass spectrometry with positive chemical ionization. J Mass Spectrom. 2001;36(7):798-805. doi: 10.1002/jms.181

16.Zhao G, Liu JF, Nyman M, Jonsson JA. Determination of short-chain fatty acids in serum by hollow fiber supported liquid membrane extraction coupled with gas chromatography. J Chromatogr B Analyt Technol Biomed Life Sci. 2007;846(1-2):202-208. doi: 10.1016/j. jchromb.2006.09.027

17. Tiihonen K, Tynkkynen S, Ouwehand A, Ahlroos T, Rautonen N. The effect of ageing with and without non-steroidal anti-inflammatory drugs on gastrointestinal microbiology and immunology. Br J Nutr. 2008;100(1):130-7. doi: 10.1017/S000711450888871X

18. Stewart ML, Nikhanj SD, Timm DA, Thomas W, Slavin JL. Evaluation of the effect of four fibers on laxation, gastrointestinal tolerance and serum markers in healthy humans. Ann Nutr Metab. 2010;56(2):9198. doi: 10.1159/000275962

19. Sauer J, Richter KK, Pool-Zobel BL. Products formed during fermentation of the prebiotic inulin with human gut flora enhance expression of biotransformation genes in human primary colon cells. Br J Nutr. 2007;97(5):928-937. doi: 10.1017/S0007114507666422

20. Francois IE, Lescroart O, Veraverbeke WS, Marzorati M, Possemiers S, Hamer $\mathrm{H}$, et al. Effects of wheat bran extract containing arabinoxylan oligosaccharides on gastrointestinal parameters in healthy preadolescent children. J Pediatr Gastroenterol Nutr. 2014;58(5):647653. doi: 10.1097/MPG.0000000000000285

21.Zheng X, Qiu Y, Zhong W, Sarah Baxter, Mingming Su, Qiong Li, et al. A targeted metabolomic protocol for short-chain fatty acids and branched-chain amino acids. Metabolomics. 2013;9(4):818-827. doi: 10.1007/s11306-013-0500-6

22. Woodrow JE, Seiber JN, Miller GC. Acrylamide release resulting from sunlight irradiation of aqueous polyacrylamide/iron mixtures. J Agric Food Chem. 2008;56(8):2773-2779. doi: 10.1021/jf703677v 